\title{
Do Financial Incentives Increase Doctors' Willingness to Publish Research? - A Pilot Study of 21 Junior Doctors
}

\section{Peter Hallas ${ }^{*}$}

\author{
*Correspondence to: Peter Hallas, Email: hallas@rocketmail.com \\ Copyright: ( 2016 by Kerman University of Medical Sciences \\ Citation: Hallas P. Do financial incentives increase doctors' willingness to publish \\ research? - A pilot study of 21 junior doctors. Int J Health Policy Manag. 2016;5(7):451- \\ 452. doi:10.15171/ijhpm.2016.54 \\ Received: 26 March 2016, Accepted: 3 May 2016, ePublished: 8 May 2016
}

\section{Dear Editor,}

As part of strategic initiatives focusing on building a research oriented culture, some healthcare institutions offer clinical staff monetary rewards for doing research. The question is if the promise of extra pay is effective as a management tool when trying to increase clinical research activity: Although it is well-documented that academics respond to the prospect of long-term financial gains, ${ }^{1,2}$ it has not been previously studied if a moderate one-time financial incentive can increase research activity of clinical staff. A proof-of-concept study was carried out to study if it was possible to develop a "supply curve for research publications" that reflected the effects of monetary incentives combined with other initiatives for facilitating research.

The study used a combination of a qualitative and quantitative approach. A convenience sample of 21 junior doctors (house officers and residents) employed at hospitals in Region Sjaelland, Denmark, participated in a semi-structured on-line survey about factors that would motivate them to do research (open-ended questions); answers were analyzed using simple content analysis; ${ }^{3}$ The three most frequent themes were:

- mentorship in the research process $(52 \%)$

- work-time allocated to research (and away from clinical work) $(43 \%)$

- a financial reward (29\%).

A new questionnaire was then constructed to quantify the relative importance of selected themes. This questionnaire contained a "virtual scenario" where the participants were asked how many research publications they thought they would publish if they were given a bonus of incremental amounts from 2000 DKK up till 75000 DKK (approximately US\$11000). The participant was also asked to what extent doing research was considered "a good career move" in their chosen specialty and if they had access to research mentorship in their current employment (Likert scale). This second survey could be answered anonymously; both questionnaires had undergone pilot testing to ensure validity. Sixteen of the participants answered the second survey. A multiple linear regression analysis was performed using a stepwise approach. ${ }^{4}$ Because of apparent heteroskedasticity for payments $>25000$
DKK this interval was excluded.

This resulted in a model for a supply curve for research publications:

$\mathrm{Q}=-17-0.1 \alpha+8.5 \log _{10} \beta+0.1 \gamma+3.3 \log _{10} \mathrm{P}$

with the parameters defined as:

$\mathrm{Q}=$ number of publications

$\mathrm{P}=$ Payment for publication

$\alpha=$ Basis salary level (dummy-variables $0=$ junior house officer; $1=$ registrar)

$\beta=$ Research as "good career move" (Likert scale; $5=$ very good)

$\gamma=$ degree of access to mentorship and departmental

research support ? (Likert scale; $5=$ very high)

$\mathrm{R}^{2}$ was 0.48 (adjusted $\mathrm{R}^{2}=0.45$ ); $F<0.05$; standard error 1.8 . Only $\gamma$ and $P$ had a $P$ value $<.05$.

The data indicates that a moderate, one-time financial incentive can be used to increase the supply of research. However, non-monetary incentives are important as monetary incentives. The finding also supports the concept that a model of "supply of research" can be used to evaluate local research incentive programs. The small sample size severely limits generalization of the results and the survey technique itself could lead to unintended biases. In additional, the coefficients in the model are determined by factors that are specific to the group examined. Even when combined, the factors identified in this study could only partly explain junior doctors' willingness to publish. Institutions considering a financial incentive should be aware of the potential problems that monetary incentives can bring to science, eg, the risk disproportionally favoring short-term endeavors. ${ }^{5}$ However, the results could be used as a starting point for further studies. Future models could be developed by incorporating the opportunity costs of doing research for clinically working doctors. ${ }^{6}$

The data of this pilot study highlights the importance of creating a research friendly culture with time allocated to research and the availability of good mentorship, rather than relying on monetary incentives alone.

\section{Ethical issues}

Not applicable.

\section{Competing interests}

Author declares that he has no competing interests.

Author's contribution

$\mathrm{PH}$ is the single author of the paper. 


\section{References}

1. Fitzenberger B, Schulze U. Up or out: research incentives and career prospects of postdocs in Germany. German Economic Review. 2014;15(2):287-328.

2. Graves PE, Marchand J, Thompson RJ. Economics departmental rankings: research incentives, constraints and efficiency. Am Econ Rev. 1982;72:1131-1141.

3. Esterberg KG. Qualitative Methods in Social Research. New York: McGraw-Hill: 2002.

4. Salvatore D. Managerial Economics in a Global Economy. New
York: Oxford University Press; 2007.

5. Geuna A. The changing rationale for European university research funding: are there negative unintended consequences? J Econ Issues. 2001;35(3):607-632. doi:10.1080/00213624.200 1.11506393

6. Embi PJ, Tsevat J. Commentary: the relative research unit: providing incentives for clinician participation in research activities. Acad Med. 2012;87(1):11-14. doi:10.1097/ acm.0b013e31823a8d99 